\title{
Applicability of Waxy Wheat Variety for Improving the Quality of Noodle and Steamed Bread
}

\author{
Liu Aifeng, Han Ran, Cheng Dungong, Li Haosheng, Cao Xinyou, Song Jianmin, Liu Cheng, \\ Guo Jun, Li Faji, Zhai Shengnan, Zi Yan, Wang Xiaolu, Liu Jianjun, Zhao Zhendong, \\ Wang Canguo
}

Crop Research Institute, Shandong Academy of Agricultural Sciences/Key Laboratory of Wheat Biology \& Genetic Improvement on North Yellow \& Huai River Valley, Ministry of Agriculture/National Engineering Laboratory for Wheat \& Maize, Jinan, China

\section{Email address:}

liuaifeng16@126.com (Liu Aifeng),395215157@qq.com (Wang Canguo)

\section{To cite this article:}

Liu Aifeng, Han Ran, Cheng Dungong, Li Haosheng, Cao Xinyou, Song Jianmin, Liu Cheng, Guo Jun, Li Faji, Zhai Shengnan, Zi Yan, Wang Xiaolu, Liu Jianjun, Zhao Zhendong, Wang Canguo. Applicability of Waxy Wheat Variety for Improving the Quality of Noodle and Steamed Bread. International Journal of Nutrition and Food Sciences. Vol. 10, No. 3, 2021, pp. 72-77. doi: 10.11648/j.ijnfs.20211003.13

Received: April 22, 2021; Accepted: May 19, 2021; Published: June 15, 2021

\begin{abstract}
Waxy wheat variety Jinuo116 is one novel type for its special starch properties bred by Crop Research Institute of SAAS (Shandong Academy of Agricultural Sciences) and released by Shandong Province. In order to fully interpret the improvement effect of waxy cultivar Jinuo116, the main food such as dry noodles, quick-frozen noodles and steamed bread were prepared by mixing Jinuo1 16 flour with different fine flour and whole meal flour, and its improving adaptability was conducted. The quality stability of Jinuo1 16 planted in six ecological zones had been analyzed firstly, the variable coefficient of grain hardness and protein content were lower $5.0 \%$, wet gluten content was $10.82 \%$. For starch pasting properties, the variable coefficient of peak time was only $1.51 \%$, other pasting properties were all lower $7.59 \%$. For dough properties, the variable coefficient of water absorption was very low (only $0.68 \%$ ), while that of stability time, weakness and Farinograph Quality Index were $22.82 \%, 12.80 \%$ and $17.95 \%$, respectively. These showed that Jinuo 116 had stable grain characteristics, dough rheological properties and starch gelatinization. Then the quality of noodles and steamed bread of mixing flour was identified by blending Jinuo116 flour into non-waxy wheat flour such as Jimai22, Jimai229, Jimai44 and Jizimai1, and showed that Jinuo116 flour could greatly improve the viscoelasticity and smoothness of dry noodles and quick-frozen noodles, and the appending proportions were changed based on the different gluten strength of non-waxy varieties, and the range of blending ratio was from $20 \%$ to $40 \%$. While considering the integrated qualities of noodles, the appropriate ratio should be controlled at about $30 \%$. Quality characteristics such as hardness, viscoelasticity, smoothness of noodles made of purple whole meal flour had been improved obviously, and the noodles had better taste quality, so the suitable proportion of flour blending was $20 \%$. The internal structure, toughness and viscosity of steamed bread made of purple whole meal flour were improved markedly, and the best internal quality characteristics were got when the adding proportion was $20 \%$. For its good quality properties especially the outstanding starch characteristics, Jinuo116 flour could be used as blending flour to improve the food quality made of non-waxy wheat.
\end{abstract}

Keywords: Waxy Wheat, Food, Quality, Applicability

\section{Introduction}

With the development of society and the diversification of market demand, waxy foods are gradually being favored by people due to their unique food characteristics, and the selection and breeding of waxy wheat varieties and their applications in the food processing industry are also gradually gaining popularity. It depends on the special starch characteristics of waxy wheat, and the special characteristics of starch are mainly reflected in the gelatinization and retrogradation characteristics of starch. Waxy wheat flour has relatively low starch gelatinization temperature and DSC measurement starting temperature, strong water holding capacity and swelling capacity, low retrogradation and aging, good freeze-thaw stability, starch transparency, swelling potential and peak viscosity are lower than those of potato, cassava and waxy corn, etc $[1,2]$, so it can be widely used in 
the development of specialty foods, the production of cold storage and quick-frozen foods. For example, adding waxy wheat flour to non-waxy wheat flour can improve the quality and taste of quick-frozen products [3]. For the gelatinization and retrogradation of waxy wheat starch are the main factors affecting high-moisture oriental traditional foods, its special starch composition plays an important role in the processing of flour products and starch. The low amylose content of waxy wheat helps to improve the quality of bread and extend the shelf life of bread [4]. Steamed bread with appropriate amount of waxy wheat flour have better flavor and can be developed as a new product [5], by blending $25.0 \%$ of waxy wheat flour can make steamed bread with better textureand maximum value of sensory score [6] and $8 \%$ of waxy wheat flour can make steamed bread with excellent taste and storage properties [7]. The addition of waxy wheat flour to non-waxy wheat flour can improve the sensory and texture properties of noodles [8-11]. Waxy wheat flour can also be used to make specialty foods such as cakes, donkey rolls, glue pudding, etc., and can replace other waxy starches in the production of pharmaceutical coatings, industrial pastes, food packaging paper, concentrates, and environmentally friendly plastics [12] Waxy wheat can also be used as a raw material additive for brewing liquor to produce liquor with a unique flavor and high liquor yield and good winemaking quality [13].

For hexaploid wheat, there is no complete waxy wheat under natural conditions, so the artificial creation is required [14]. Jinuo116 is cultivated by using conventional hybridization and recrossing combined with molecular marker assisted breeding technology and supplemented by iodine-potassium iodide grain dyeing method. The three waxy genes in hexaploid wheat endosperm have a dose effect on the synthesis of amylose, and their effects on amylose content are different [15-17]. The deletion of the Wx-B1 subunit gene or substitution has the greatest impact on amylose content, and the deletion of Wx-A1 and Wx-D1 subunit genes has less impact [18]. Only when these three pairs of genes are homozygous or deleted at the same time can they become a complete waxy mutant, namely waxy wheat [19]. The WX protein molecular marker detection showed that the WX-A1, B1, and D1 subunits of Jinuo 116 are all deletion mutants, with amylopectin content of $100 \%$, and it is a whole waxy wheat. The research is based on the unique excellent starch quality characteristics of Jinuo116, and explores the best blending ratio to improve food quality by adding different proportions of flour to provide a certain theoretical support for giving full play to the improvement effect of Jinuo 116 in food processing.

\section{Materials and Methods}

\subsection{Experiment Materials}

Jinuo116, Jizimai1, Jimai22, Jimai44 and Jimai229 were selected as experimental materials. Jinuo 116 was planted in 6 trial points including Jinan, Yantai, Dezhou, Tai'an, Jining and Heze from year 2017 to 2019, grain were harvested, and will be used for quality stability analysis and flour blending analysis. Jizimai1, Jimai22, Jimai44 and Jimai229 are planted in Jinan trial point during year 2018-2019, and the grain were harvested to be used to analyze the food quality adaptability by blending Jinuo116.

\subsection{Experiment Methods}

Whole wheat meal of Jizimail was prepared by superfine whole powder milling process technology. The refined flour of Jinuo116, Jimai22, Jimai44 andJimai229 are made by Buhler mill. Jinuo116 flour is mixed with Jizimai1 whole meal flour in a ratio of $0,10,20$, and $30 \%$ for making steamed bread. Mix Jinuo116 flour with Jizimai1 whole meat flour, Jimai22 flour, Jimai44 flour and Jimai229 flour in the proportions of 0 , $10,20,30,40,50,60,70,80,90,100 \%$ to make noodles. Infratec 1241 type Flowserve Near Infrared analyzer was used to measure the protein content of grains. SKCS4100 single-grain characteristics system was used to measures the hardness of grains. GM2200 gluten meter was used tomeasure the wet gluten content according to the GB5506-85 method. Brabender Farinograph was used to determine the characteristics of dough quality. The fast viscometer formulated by AACC76-21 was used to determine starch gelatinization properties. The processing and quality evaluating of noodles and steamed bread are according to the GB/T17320-2013 method.

\subsection{Data Processing}

Adopt SPSS and Excel system.

\section{Results and Analysis}

\subsection{Quality Characteristics and Stability Analysis of Jinuo116}

The quality indicators of Jinuo116 in the six ecological regions are represented by the average value and the variation coefficient in table 1 . The grain hardness is 48.78 and grain protein content on dry basis is $14.36 \%$, and the variation coefficients are both below $5.0 \%$. The wet gluten content is $34.84 \%$, and its variation coefficient is $10.82 \%$. So it indicates that its grain is soft, and its grain characteristics have good stability. For the dough characteristics, the water absorption is as high as $78.68 \%$, its variation coefficient is only $0.68 \%$, while the stability time is short, the weakening value is large, the farinograph quality number (FQN) is low, and their variation coefficients are all high, which indicates that the dough rheological properties of Jinuo116 are poor and is relatively easy to be affected by the environment. For starch gelatinization characteristics, the largest variation coefficient among the various indicators is only $7.59 \%$, the setback value is $284.0 \mathrm{cP}$, and its variation coefficient is $6.11 \%$, which indicates that the starch gelatinization characteristics are not easily affected by the ecological environment. In summary, Jinuo116 has good grain characteristics and starch gelatinization properties with good stability in the six ecological regions. 
Table 1. Quality Characteristics of Jinuo116.

\begin{tabular}{llllllll}
\hline Qulity Index & $\begin{array}{l}\text { Grain } \\
\text { hardness }\end{array}$ & $\begin{array}{l}\text { Protein content/ } \\
\text { dry bases\% }\end{array}$ & $\begin{array}{l}\text { Wet gluten } \\
\text { content/14\% }\end{array}$ & $\begin{array}{l}\text { Water } \\
\text { absorption/\% }\end{array}$ & $\begin{array}{l}\text { Stability } \\
\text { time /min }\end{array}$ & $\begin{array}{l}\text { Weakness/ } \\
\text { FE }\end{array}$ & $\begin{array}{l}\text { Farinograph } \\
\text { quality number }\end{array}$ \\
\hline Average & 48.78 & 14.36 & 34.84 & 78.68 & 2.60 & 156.00 & 63.67 \\
Variation coefficient $\%$ & 4.78 & 4.00 & 10.82 & 0.68 & 22.82 & 12.80 & 17.95 \\
\hline
\end{tabular}

Table 1. Continued.

\begin{tabular}{llllllll}
\hline Qulity Index & $\begin{array}{l}\text { Pasting } \\
\text { temperature/ }{ }^{\circ} \mathbf{C}\end{array}$ & $\begin{array}{l}\text { Peak viscosity / } \\
\mathbf{c P}\end{array}$ & $\begin{array}{l}\text { Trough } \\
\text { viscosity/cP }\end{array}$ & Breakdown /cP & $\begin{array}{l}\text { Final } \\
\text { viscosity } / \mathbf{c P}\end{array}$ & $\begin{array}{l}\text { Setback } \\
/ \mathbf{c P}\end{array}$ & $\begin{array}{l}\text { Peak time } \\
/ \mathbf{m i n}\end{array}$ \\
\hline Average & 66.73 & 2040.33 & 933.33 & 1107.00 & 1217.33 & 284.00 & 3.87 \\
Variation coefficient $/ \%$ & 0.54 & 7.34 & 7.44 & 7.59 & 6.67 & 6.11 \\
\hline
\end{tabular}

\subsection{Analysis on Food Quality Adaptability of Jinuo116 Blended Flour}

\subsubsection{Effect on the Quality of Different Types of Noodles}

Due to the special starch properties of Jinuo116, its noodles are sticky and slippery, though it is too soft to be used alone to make noodles. While it can be added as a powder to common wheat flour and purple whole wheat meal to improve the taste of noodles, and also be used to improve the quality of quick-frozen noodles after re-cooking for its excellent freeze-thaw stability.

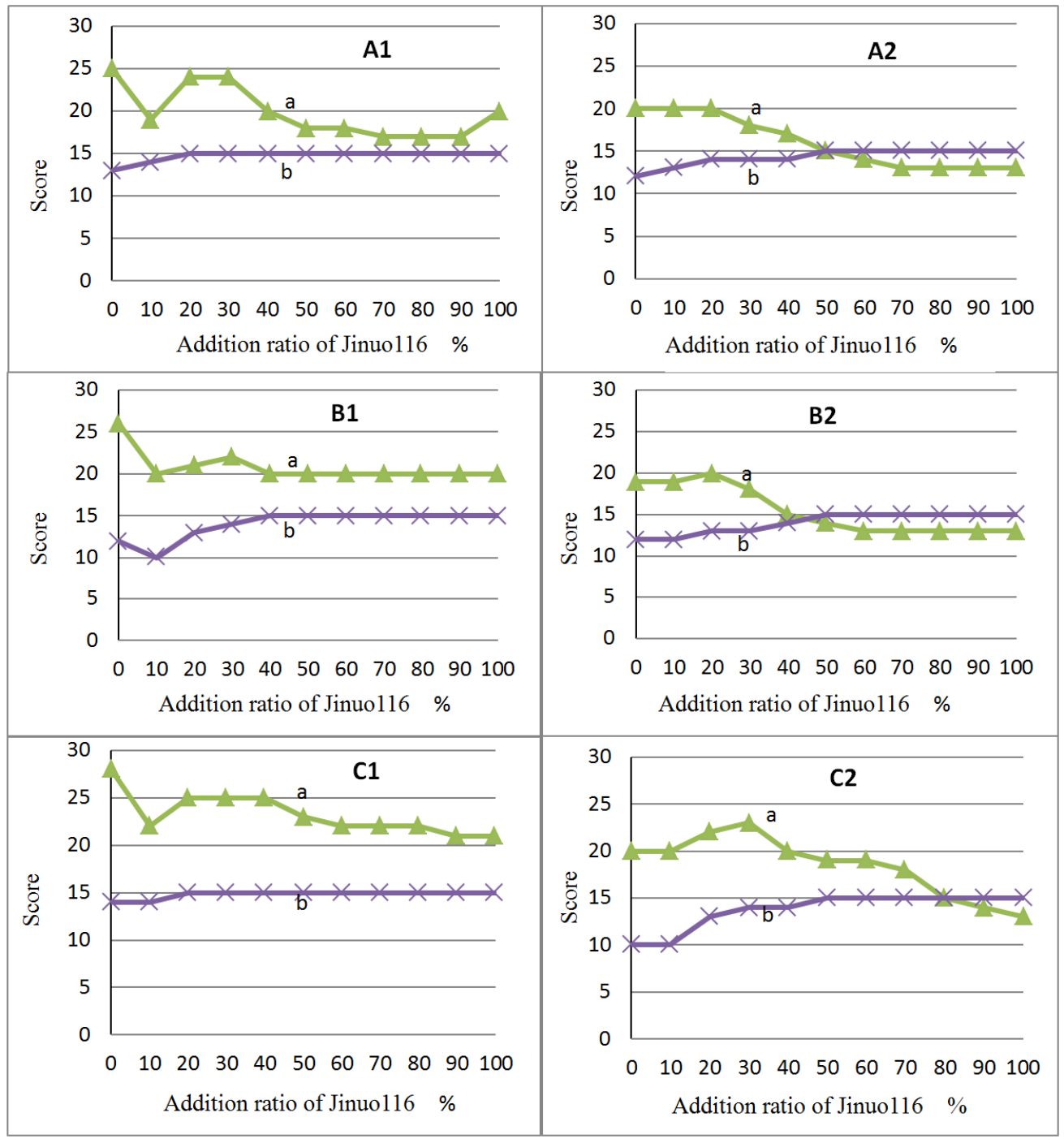

Figure 1. Effect of blended Jinuo116 flour on the viscoelasticity and smoothness of noodles.

A1: Dry noodles of Jimai22; A2: Quick-freeze noodles of Jimai22; B1: Dry noodles of Jimai44; B2: Quick-freeze noodles of Jimai44; C1: Dry noodles of Jimai229; C2: Quick-freeze noodles of Jimai229. a: Viscoelasticity; b: Smoothness 
The effect of waxy wheat on the quality of common wheat flour noodles is mainly manifested in viscoelasticity and smoothness (Figure 1). With the increase of the proportion of Jinuo116 flour, the effect on the viscoelasticity score shows a trend of first decline and then increase of dry noodles. When the addition ratio is $10 \%$, the viscoelasticity scores of the noodles made of three types wheat flour (Jimai22 Jimai44 and Jimai229) are all lower than that of the original flour. When the addition ratio is $20 \%$, the viscoelasticity scores all increase significantly. When the addition ratio is greater than $30 \%$, the viscoelasticity score shows a downward trend. The smoothness score shows a gradual upward trend with the increase of the proportion of addition. When the proportion of addition reaches $20 \%$, the smoothness score of Jimai22 and Jimai229 noodles reaches the highest score (15 points), when the proportion of addition reaches $40 \%$, Jimai44 noodles scored the highest in smoothness. All these show that the addition of Jinuo116 flour improves the smoothness of the noodles, though there is certain difference in the addition ratio for noodles made of different type variety. The appropriate ratio of Jinuo116 flour blended to flour of Jimai22 and Jimai229 can be controlled at 20-30\%, and $30-40 \%$ to flour of Jimai44. For quick-frozen noodles, when the addition ratio of Jinuo 116 flour is less than $20 \%$, the viscoelasticity scores of Jimai22 mixed flour noodles and Jimai44 mixed flour noodles both change little., while when the addition ratio is higher than $30 \%$, theviscoelasticityscore decreased sharply. The viscoelasticity score of Jimai229 mixed flour noodles increased firstly and then decreased with the increase of the addition ratio of Jinuo 116 flour, and when the addition ratio was $30 \%$, the viscoelasticity score was the highest, and then quickly decreased. The smoothness score is increasing with the increase addition ratio, and the score is close to the highest when the proportion of addition is $40 \%$. Combined with the viscoelasticity and smoothness of the quick-frozen noodles, for Jimai22 and Jimai44, the appropriate addition ratio of Jinuo116 can be controlled within 20-30\%, and for Jimai229, the appropriate addition ratio can be controlled within 30-40\%. To sum up, the addition of Jinuo116 flour can significantly improve the tasting quality of dry noodles and quick-frozen noodles, such as viscoelasticity and smoothness, although there are certain differences in the addition ratio of varieties with different gluten strength. Considering the comprehensive quality characteristics of noodles, the appropriate addition ratio of Jinuo 116 should be controlled at about $30 \%$.

The effect of waxy wheat flour on the quality of purple whole wheat meal noodles is mainly manifested in hardness, viscoelasticity and smoothness (Figure 2). Purple whole wheat meal noodles score all are low in hardness, viscoelasticity and smoothness, and it tastes poor andrough, while waxy wheat flour noodles score is low in hardnessand high in smoothness and viscoelasticity. By blending waxy wheat flour into Purple whole wheat meal to improve mixed noodles quality, with the increase in the amount of waxy wheat flour added, the scores of the three indicators of the mixed flour noodles all show an increasing trend. Among them, the scores of hardness and viscoelasticity get the highest when the addition ratio is $20 \%$, and then become smaller. All these show that the addition of waxy wheat flour to purple whole wheat meal improves the hardness, viscoelasticity and smoothness of noodles, making the taste quality of the noodles significantly better than that of purple whole wheat meal noodles.

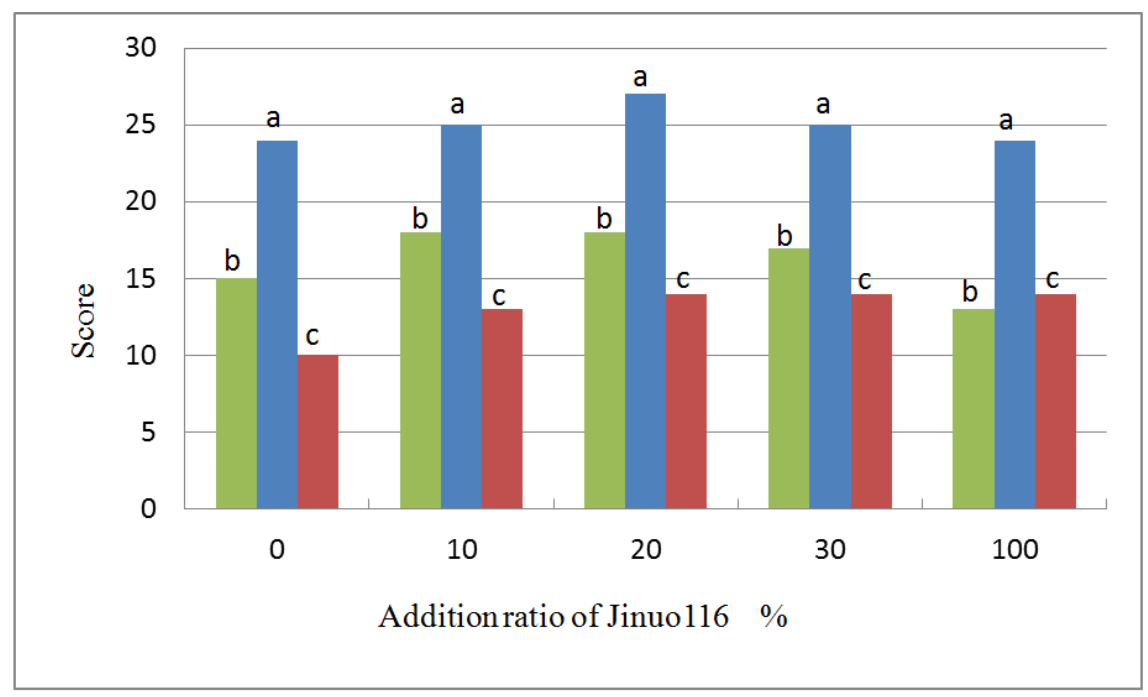

a: Viscoelasticity; b: Hardness; c: Smoothness

Figure 2. Effect of blended Jinuo116 flour to purple whole wheat meal on the viscoelasticity, hardness and smoothness of noodles.

\subsubsection{Effect on the Quality of Steamed Bread Made of Purple Wheat Whole Flour}

The steamed breadmade of purple wheat whole flour has a light purple color, and the external quality traits such as skin structure and elasticity are the same as those of steamed bread made of high-quality wheat flour, but the internal quality traits such as structure, toughness and stickiness are inferior (to be published separately). After be blended with waxy wheat flour, the quality of steamed bread has changed 
significantly (Table 2). With the increasing proportion of Jinuo116 flour, the specific volume, specific-volume score, and width/height ratio all become larger, while the score of width/height ratio becomes smaller, which indicates that the volume increases and the shape becomes slightly flat. When the addition ratio is $30 \%$, these four quality indicators are all worse, and the shape is flat, the skin structure score and elasticity score both increase, which indicates that the skin structure becomes smoother and more elastic. And the score of internal structure increases, the score of toughness and viscosity are increasing when the addition ratio below $20 \%$, while the score decreases when the addition ratio reaches $30 \%$, which indicates that when the addition ratio is less than $20 \%$, the internal structure gradually becomes delicate with the increase of the addition ratio, while the addition ratio reaches $30 \%$, the internal structure becomes more sticky. In summary, the suitable addition ratio of waxy wheat flour to improve the quality of steamed bread made of purple whole wheat meal can be controlled within $10-20 \%$.

Table 2. Effect of Blended Jinuol16 Flour on the Steamed Bread Quality of Purple Whole Wheat meal.

\begin{tabular}{|c|c|c|c|c|c|c|c|c|c|c|c|c|c|}
\hline \multirow[b]{2}{*}{$\begin{array}{l}\text { Ratio } \\
/ \%\end{array}$} & \multirow{2}{*}{$\begin{array}{l}\text { Specific } \\
\text { volume/ml/g }\end{array}$} & \multirow{2}{*}{$\begin{array}{l}\text { Width/height } \\
\text { ratio }\end{array}$} & \multicolumn{11}{|c|}{ Score of Steamed-bread } \\
\hline & & & $\begin{array}{l}\text { Specific } \\
\text { volume }\end{array}$ & Width/height & Shape & Color & $\begin{array}{l}\text { Skin } \\
\text { structure }\end{array}$ & Elasticity & $\begin{array}{l}\text { Section } \\
\text { structure }\end{array}$ & Toughness & Viscosity & Flavor & $\begin{array}{l}\text { Total } \\
\text { score }\end{array}$ \\
\hline 0 & $1.65 \mathrm{a}$ & $1.60 \mathrm{a}$ & $3.46 \mathrm{a}$ & $4.02 \mathrm{a}$ & $8 \mathrm{a}$ & $8 \mathrm{a}$ & $6 a$ & $5 a$ & $11 \mathrm{a}$ & $6 a$ & $7 \mathrm{a}$ & $4 a$ & $62.48 \mathrm{a}$ \\
\hline 10 & $1.84 \mathrm{a}$ & $1.70 \mathrm{a}$ & $5.37 \mathrm{a}$ & $2.97 \mathrm{a}$ & $7 \mathrm{a}$ & $8 \mathrm{a}$ & $8 \mathrm{a}$ & $7 \mathrm{a}$ & $12 \mathrm{a}$ & $7 \mathrm{a}$ & $8 \mathrm{a}$ & $4 \mathrm{a}$ & $69.34 \mathrm{c}$ \\
\hline 20 & $1.72 \mathrm{a}$ & $1.77 \mathrm{a}$ & $4.18 \mathrm{a}$ & $2.35 \mathrm{a}$ & $7 \mathrm{a}$ & $8 \mathrm{a}$ & $8 \mathrm{a}$ & $7 \mathrm{a}$ & $12 \mathrm{a}$ & $8 \mathrm{a}$ & $8 \mathrm{a}$ & $4 a$ & $68.53 b c$ \\
\hline 30 & $1.67 \mathrm{a}$ & $1.79 \mathrm{a}$ & $3.70 \mathrm{a}$ & $2.16 \mathrm{a}$ & $6 a$ & $8 \mathrm{a}$ & $8 \mathrm{a}$ & $8 \mathrm{a}$ & $12 \mathrm{a}$ & $7 \mathrm{a}$ & $6 a$ & $4 \mathrm{a}$ & $64.86 \mathrm{ab}$ \\
\hline
\end{tabular}

Note: Different letters indicate a significant difference of $5 \%$.

\section{Discussion}

Waxy wheat has a broad application prospect. Its flour used as a raw material for "modified" starch foods can adjust the amylose content and improve the quality of food. It will play an important role in changing the structure and composition of diet of resident [20]. Waxy wheat flour could improve the sensory and texture properties of noodles [8-11]. In this study, the addition of Jinuo116 flour improved the tasting quality of non-waxy wheat dry noodles, such as viscoelasticity and smoothness. Andthe addition ratio varied with the strength of gluten. To measure the overall quality characteristics of the noodles, the appropriate addition ratio of Jinuo116 flour could be controlled at about $30 \%$. For noodles made of purple wheat whole meal, its tasting quality such as hardness, viscoelasticity and smoothess were poor. While with the addition of Jinuo116 flour, the noodles score of hardness, viscoelasticity and smoothness increased, and the tasting quality became better. Waxy wheat flour also had a good effect on improving the taste and storage performance of steamed bread [5-7]. In this study, Jinuo116 flour could improve the shape, skin structure, taste and internal structure of steamed bread made of purple wheat whole meal. Especially because waxy starch had higher water holding capacity, lower setting and aging speed, it could increase the fresh-keeping ability of flour products, so itwas suitable for making cold storage and quick-frozen food [1, 4, $21,22]$. In this study, it was also confirmed that the addition of Jinuo116 flour could improve the tasting qualities such as viscoelasticity and smoothness of quick-frozen noodles made of common wheat. At the same time, the application of Jinuo116 in cold storage and quick-frozen food needed to be further explored.

\section{Conclusion}

Waxy wheat variety Jinuo116 is one novel type for its special starch properties bred by Crop Research Institute of
SAAS. The study found that Jinuo116 has good grain characteristics and starch gelatinization properties with good stability. In addition, it found that the flour of Jinuo116 could greatly improve the viscoelasticity and smoothness of dry noodles and quick-frozen noodles. For its good quality properties especially the outstanding starch characteristics, Jinuol16 flour could be used as blending flour to improve the food quality made of non-waxy wheat.

\section{Acknowledgements}

This research was supported by Agricultural Variety Improvement Project of Shandong Province (2019LZGC001), Key R\&D Program of Shandong Province (Major Technological Innovation Project) (2020CXGC0108053).

\section{References}

[1] Abdel-Aal E S M, Hucl P, Chibbar $R$ N, et al. Physicochemical and structural characteristics of flours and starches from waxy and nonwaxy wheat $[\mathrm{J}]$. Cereal Chemistry, 2002, 79 (3): 458-464.

[2] Zhang Xiao, Gao Derong, Lu Guofeng, et al. Comparison of the Starches Properties of Waxy Wheat and Other Crops [J]. Scientia Agricultura Sinica, 2013, 46 (11): 2183-2190.

[3] Yang Yanfang, Zhu Kexue, Guo Xiaona, et al. Effect of Waxy Wheat Flour on Properties of Flour Mixture and Freeze-thaw Stability of Dough [J], Modern Flour Milling Industry, 2014, 03: 23-28.

[4] Liu Aifeng, Song Jianmin, Zhao Zhendong, et al. Effects of Waxy Flour Blending on Dough Rheological Properties and Bread-making Quality of Non-waxy Flour [J]. Scientia Agricultura Sinica, 2004, 37 (6): 902-907.

[5] Su Dongmin, Chen Chen, Wei Xueqin, et al. Effects of Waxy Wheat Flour Blending on Rheological Properties of Dough and Quality of Mantou [J]. Journal of Henan University of Technology (Natural Science Edition), 2008, 29 (2): 1-7. 
[6] Zhang Huanxin, Zhang Wei, Xu Chunzhong. Effect of Waxy Wheat Flour on Rheological Properties of Flour Blending and Steamed Bread Quality [J]. Food Science, 2014, 35 (3): 80-84.

[7] Guo Kexue, Ding Wenping. Study on Physicochemical Properties of Waxy Wheat Flour and Its Application in Steamed Bread [J]. Grain and Oil Processing and Food Machinery, 2005, 12: 69-71.

[8] Qin PengMa Chuanxi, Wu Ronglin, et al. Effect of Waxy Wheat Flour Blending on Quality of Dry White Chinese Noodles [J]. Journal of the Chinese Cereals and Oils Association, 2008, 23 (3): 17-23.

[9] Chen Dongsheng, C. Kiribuchi-Otobe, XU Zhaohua, et al. Effect of Wx-A, Wx-B and Wx-D Protein on Starch Properties and Chinese Fresh Noodle Quality [J]. Scientia Agricultura Sinica, 2005, 38 (5): 865-873.

[10] Liang Rongqi, Yang Fengping1, Su Qing, et al. Effects of Waxy Flour Blending on Starch Properties and Noodle Quality of Non-waxy Flour [J]. Acta Agriculturae Boreali-sinica, 2007, 3: 16-20.

[11] Liu Aifeng, Wang Canguo, Cheng Dungong, et al. Quality Characteristics of Waxy Wheat Flour Blending with Non-waxy Wheat Flour and Its Effects on Noodle Making Quality [J]. Food Science, 2017, 38 (3): 94-100.

[12] Sun Lian, Sun Hui. Research Progress on the Properties of Glutinous Wheat Flour $[\mathrm{J}]$. Grain, Oil and Food Science and Technology, 2008, 1: 1-4.

[13] Zhao Guojun, Li Bin, Xu Zhibin, et al. Effect of Waxy Wheat on the Properties of Xiaoqu Liquor Brewed with Common Wheat and Non-glutinous Sorghum [J]. Journal of Triticeae Crops, 2013, 33 (5): 942-945.

[14] Kiribuchi O C, Nagamine T, Yanagisawa T, et al. Production of Hexaploid Wheats with Waxy Endosperm Character [J]. Cereal Chemistry, 1997, 74 (1): 72-74.

[15] Miura H, Tanii S. Endosperm Starch Properties in Several Wheat Varieties Preferred for Japanese Noodles [J]. Euphytica, 1994, 72: 171-176.
[16] Miura H, Sugawara A. Do Sage Effects of the Three Wx Genes on Amylose Synthesis in Wheat Endosperm [J]. Theoretical and Applied Genetics, 1996, 93 (7): 1066-1070.

[17] Ainsworth C, Clark J, Balsdon J. Expression, Organization and Structure of the Genes Encoding the Waxy Protein (granule-bound starch synthase) in Wheat [J]. Plant Molecular Biology, 1993, 22 (1): 67-82.

[18] Wang Zining, Zhang Yanmin, Guo Beihai, etal. Waxy Wheat Production by Haploid Plant Production [J]. Acta Agriculturae Boreali-Sinica, 2001, 16 (2): 53-57.

[19] Yao Jinbao, Yang Xueming, Yao Guocai, et al. Research Progress on Waxy Wheat in China [J]. Journal of Plant Genetic Resources, 2004, 5 (2): 201-204.

[20] OU Qiaoming, NI Jianfu, YE Chunlei. Research Progress in Waxy Wheat and Wx Genes [J]. Henan Agricultural Sciences, 2006 (1): 23-28.

[21] Graybosch R A. Waxy Wheat: Origin, Properties, and Prospects [J]. Trends Food Sci Technol, 1998, 8 (4): 135-142.

[22] Jinhee Yi, Willian L. Kerr and Jerry W. Johnson. Effects of Waxy Wheat Flour and Water on Frozen Dough and Bread Properties [J]. Journal of Food Science, 2009, 74 (5): 278-284.

\section{Biography}

Liu Aifeng (1969-), female, Qingzhou, Shandong, researcher, $\mathrm{PhD}$, mainly engaged in the cultivation of new wheat varieties, the food quality improvement of high-quality wheat and special-use wheat, and the research and development of high-end food. E-mail: liuaifeng16@126.com

Wang Canguo (1985-), male, Taian, assistant researcher, master's degree, wheat quality measurement and improvement of food quality wheat research specialties. 